\title{
FORMACIÓN INICIAL DOCENTE PARA LA EDUCACIÓN INCLUSIVA. ANÁLISIS DE TRES PROGRAMAS CHILENOS DE PEDAGOGÍA EN EDUCACIÓN BÁSICA QUE INCORPORAN LA PERSPECTIVA DE LA EDUCACIÓN INCLUSIVA
}

\author{
Constanza San Martín ${ }^{1}$ \\ Cristóbal Villalobos ${ }^{2}$ \\ Carla Muñoz ${ }^{3}$ \\ Ignacio Wyman ${ }^{4}$
}

\begin{abstract}
RESUMEN
El presente artículo indaga en la importancia de la educación inclusiva en la formación inicial docente en tres programas de Pedagogía en Educación Básica del sistema de educación superior chileno, que incorporan elementos de la educación inclusiva en sus descripciones y perfiles de egreso. Específicamente, la investigación busca conocer cómo se entiende la educación inclusiva en el diseño curricular de estos programas y de qué manera estos incluyen esta dimensión dentro de los procesos formativos que llevan a cabo. A través de una metodología de métodos mixtos, se realiza un análisis de los perfiles de egreso y de las mallas curriculares de cada programa, que se contrastan con los discursos de actores clave de cada uno de ellos. Los resultados muestran que la mayoría desarrolla una aproximación hacia la educación inclusiva basada en el discurso por sobre la implementación de prácticas pedagógicas concretas y en una incorporación parcializada y específica en algunas asignaturas. Adicionalmente, no existen altos niveles de coherencia interna respecto de qué se entiende por educación inclusiva y cómo esta se debe desarrollar para la formación inicial del profesorado en los programas analizados.
\end{abstract}

Palabras clave: Chile, educación inclusiva, formación inicial docente, métodos mixtos.

Facultad de Educación, Universidad Diego Portales, Santiago, Chile. Contacto: constanza. sanmartin@mail.udp.cl

2 Centro de Estudios de Políticas y Prácticas en Educación, Pontificia Universidad Católica de Chile, Santiago, Chile. Contacto: clvillal@uc.cl

3 Centro para la Transformación Educativa, Pontificia Universidad Católica de Chile, Santiago, Chile.Contacto: clmunozl@uc.cl

4 Centro de Estudios de Políticas y Prácticas en Educación, Pontificia Universidad Católica de Chile, Santiago, Chile. Contacto: iawyman@uc.cl 


\title{
PRE-SERVICE TEACHER TRAINING FOR INCLUSIVE EDUCATION. ANALYSIS OF THREE CHILEAN PROGRAMS OF PEDAGOGY IN ELEMENTARY EDUCATION THAT ENFASIZE INCLUSIVE EDUCATION
}

\author{
ABSTRACT
}

This article explores the importance of inclusive education in pre-service training by examining three academic programs focused on Pedagogy in Elementary Education in the Chilean higher education system that incorporate elements of inclusive education in their descriptions and profiles. Specifically, the research seeks to understand how to inclusive education is defined in the curriculum of these programs and how they include this dimension in the educational training of these students. Using mixed methods approach, the study analyzes the professional profiles and the curricula of each academic program. This analysis is contrasted with the discourse of directors and teachers from each of the programs. The results show that the most programs develop an approach towards inclusive education is based more heavily in theoretical discourse rather than in the implementation of specific teaching practices. Furthermore, the results show that programs incorporate the concept of inclusive education only in relation to specific subjects, without developing a transversal vision of the topic. Additionally, the study did not find high levels of internal consistency regarding what is meant by inclusive education and how this should be developed for initial training of teachers in the programs analyzed.

Keywords: Chile, inclusive education, mixed method, pre-service Training.

\section{Introducción}

Desde mediados del siglo pasado los sistemas educativos de todo el orbe han aumentado — con distintas intensidades y a través de diversos mecanismos - su capacidad de integrar a más estudiantes. Una de las consecuencias más importantes de este proceso es que las escuelas se han convertido progresivamente en espacios más diversos y complejos para desarrollar procesos de enseñanza. Así, la convergencia de niños, niñas y adolescentes de diferente origen social, étnico, racial, país de procedencia o capacidades físicas, sensoriales e intelectuales desafía una de las principales tareas de las comunidades escolares: lograr que todos los estudiantes participen y aprendan del proceso de aprendizaje. Las disparidades en resultados académicos y habilidades psicosociales y la prevalencia de hitos disruptivos en la trayectoria escolar de poblaciones minoritarias o subordinadas no hacen más que agudizar el diagnóstico (Glick, Yabiku \& Bates, 2008; Román \& Perticará, 2012). 
22 FORMACIÓN INICIAL DOCENTE PARA LA EDUCACIÓN INCLUSIVA. ANÁLISIS DE TRES PROGRAMAS CHILENOS DE PEDAGOGÍA EN EDUCACIÓN BÁSICA QUE INCORPORAN LA PERSPECTIVA DE LA EDUCACIÓN INCLUSIVA - C. San Martín, C. Villalobos, C. Muñoz y I. Wyman

En el caso de Chile, la temática de integración escolar y, posteriormente, de la educación inclusiva ha estado presente en la discusión nacional desde el retorno a la democracia. La principal forma en que se ha buscado instalar este discurso en el país ha sido por medio de la creación de una serie de normativas, políticas y orientaciones técnico-pedagógicas, desarrolladas principalmente por el Estado (Ministerio de Educación de Chile, Mineduc, 2005; Mineduc, 2007; Mineduc, 2015). De esta forma, se ha promovido el reconocimiento de la diversidad de estudiantes en el sistema escolar, buscando generar lineamientos para la implementación de medidas y acciones concretas que permitan proporcionar apoyos pertinentes para responder a las necesidades educativas dentro de las escuelas y salas de clases. Así, se han realizado esfuerzos por llevar a la práctica una de las formas compartidas internacionalmente de entender la inclusión educativa, al considerarla como:

un proceso para abordar y responder a la diversidad de necesidades de todos los niños, jóvenes y adultos mediante el aumento de la participación en el aprendizaje, las culturas y las comunidades, y la reducción y eliminación de la exclusión dentro y desde la educación (United Nations Educational, Scientific and Cultural Organization, Unesco, 2009, p. 8).

La definición anterior se aleja del entendimiento tradicional de la educación inclusiva como aquella que se dirige a grupos específicos de estudiantes, trasladándose a una comprensión más compleja, sustentada en la convicción de que la responsabilidad del sistema educativo regular es proporcionar oportunidades de aprendizaje de calidad para todos. No obstante, y a pesar de la importancia de esta definición, las políticas educativas chilenas se han desarrollado principalmente desde una perspectiva tradicional de la inclusión, circunscribiéndola a temáticas específicas como los estudiantes con necesidades educativas especiales o los estudiantes de origen étnico, sin considerar integralmente la necesidad de incorporar las diferencias sociales, culturales, políticas y académicas en el proceso de enseñanza (Infante, 2010; Infante \& Matus, 2009; Jiménez y Montecinos, 2015; Tenorio, 2005; 2011). 
Una de las estrategias internacionalmente reconocidas para potenciar la perspectiva de la educación inclusiva en los sistemas escolares está relacionada con la capacidad de los estados de contar con docentes capacitados para trabajar y desenvolverse en salas de clases con un alto grado de diversidad (Darling-Hammond \& Baratz-Snowden, 2007). La literatura internacional ha indicado que el no contar con un currículo que promueva el desarrollo de prácticas pedagógicas inclusivas contribuye a perpetuar la segregación escolar, al disminuir las oportunidades de participación, aprendizaje y progreso de todos los estudiantes en el sistema escolar (Florian, Young \& Rouse, 2010). Además, la ausencia de una formación de profesores inclusivos promovería y perpetuaría una visión del ser humano centrada en el utilitarismo, donde se acepta que personas pertenecientes a algunas minorías se sientan excluidas o sean perjudicadas por el sistema educativo (Lewin, 2014).

En Chile, sin embargo, la calidad de la formación docente y su nivel de comprensión y utilización de la perspectiva de la educación inclusiva están actualmente puestos en tela de juicio por diversos motivos. En primer lugar, los resultados obtenidos por evaluaciones que miden el conocimiento de los futuros profesores muestran importantes deficiencias en sus resultados, tanto a nivel nacional (Prueba Inicia) como a nivel internacional (TEDS-M) (Tatto et al., 2012). A partir de estas mediciones, varios investigadores han concluido que los egresados de Pedagogía en Chile no están totalmente preparados para enfrentar la diversidad que existe en el aula y además tienen un aprendizaje comparativamente menor al de otros países (Ávalos y Matus, 2010; Ávalos, Navarro y Téllez, 2010; Sotomayor, Parodi, Coloma, Ibáñez y Cavada, 2011) siendo esta, además, una de las principales debilidades reconocidas por los propios docentes (Rufinelli, 2013).

En segundo término, se ha constatado que la oferta de programas de Pedagogía ha crecido excesivamente en los últimos diez años, lo que ha generado como consecuencia una alta precarización y estratificación del sistema de formación docente (Bellei y Valenzuela, 2010; Berner y Bellei, 2011; Cox, Meckes y Bascopé, 2010). Esto ha provocado una distribución inequitativa de los nuevos docentes en el 
24 FORMACIÓN INICIAL DOCENTE PARA LA EDUCACIÓN INCLUSIVA. ANÁLISIS DE TRES PROGRAMAS CHILENOS DE PEDAGOGÍA EN EDUCACIÓN BÁSICA QUE INCORPORAN LA PERSPECTIVA DE LA EDUCACIÓN INCLUSIVA - C. San Martín, C. Villalobos, C. Muñoz y I. Wyman

sistema escolar, puesto que aquellos profesores que fueron formados en instituciones que tienen mejores procesos de selección y que son consideradas de mayor calidad "no enseñan en los establecimientos escolares que más necesitarían de ellos" (Ávalos, 2014, p. 12). No es ilógico pensar, entonces, que la capacidad de reconocer y trabajar con la diversidad también está desigualmente distribuida entre los profesores que ejercen la profesión en el sistema escolar del país.

Finalmente, y aunque no existe mayor evidencia respecto de los procesos formativos de los estudiantes de los programas de Pedagogía en Chile, algunos autores señalan que si bien desde las políticas educativas se ha presionado a las distintas universidades e instituciones de formación docente para generar respuestas concretas a la diversidad del alumnado (Mineduc, 2007), la mayoría de los programas y el currículo de educación superior para profesores incluye herramientas a nivel de metodologías y didácticas que se centran en el aprendizaje de un alumno promedio, sin considerar la diversidad del estudiantado (Infante, 2010; Infante y Matus, 2009; Tenorio, 2011), entendiendo la escuela como un espacio homogéneo, donde el estudiante debe cumplir con estándares tradicionales y organizados en torno a un ideal normativo que no existe en la realidad (Infante, 2010; Jiménez y Montecinos, 2015).

Considerando estos elementos, la presente investigación buscar conocer de qué manera tres programas de Pedagogía en Educación Básica (PEB) del sistema de educación superior chileno, que incorporan elementos de la educación inclusiva en sus descripciones y perfiles de egreso (Villalobos, San Martín y Wyman, 2016) le otorgan importancia al enfoque educativo inclusivo dentro de su currículo formativo. Específicamente, el estudio busca responder a las siguientes preguntas: ¿de qué forma se incorpora la educación inclusiva en la formación inicial de docentes de educación básica?, ¿en qué momento de los programas se incorporan los elementos de la educación inclusiva? y, ¿bajo qué conceptos se entiende la idea de la educación inclusiva? Para ello, se realiza un análisis de las mallas curriculares y perfiles de egreso distinguiendo los tipos de asignaturas asociadas a la temática, su ubicación temporal en la malla curricular y la frecuencia de conceptos alusivos a la educación inclusiva, los que 
se contrastan con los discursos y representaciones de actores clave (directores de carrera y docentes de las asignaturas) de cada uno de los programas.

\section{Antecedentes}

La literatura nacional e internacional que relaciona la formación inicial docente con la diversidad escolar es amplia y profusa (European Agency for Development in Special Needs Education, 2011; Infante, 2010; Melnick \& Zeichner, 1995; Rufinelli, 2013).

En general, los estudios realizados se han focalizado en dos aspectos para explicar esta relación: por una parte, las características de origen de los estudiantes de Pedagogía y, por otra, las características de los programas de formación.

Respecto del primer aspecto, se ha destacado que no ha habido una mayor diversificación de las características de origen de los docentes y quienes se forman para serlo. Como destacan Melnick y Zeichner (1995), un nodo importante en la discusión por la preparación de docentes para enfrentar la diversidad en aula es el "problema de la selección", que alude a la posesión de características homogéneas de los estudiantes que se forman como docentes, las que muchas veces se alejan de las características y experiencias de vida del sistema escolar (Darling-Hammond \& Baratz-Snowden, 2007; Melnick \& Zeichner, 1995). Además, algunos autores destacan que los profesores tienden a sobreestimar su perspectiva y a subestimar la del estudiante y su entorno (Darling-Hammond \& Baratz-Snowden, 2007; De Jong \& Harper, 2005; Liu \& Milman, 2010; Organization for Economic Cooperation and Development, OECD, 2010), lo que constituye un aspecto crítico para el desarrollo de procesos inclusivos en el aula. En el caso chileno, también existe una homogenización del origen de los docentes, pues la mayoría de los estudiantes que ingresan a las carreras de Pedagogía Básica y Educación Parvularia tienen una base formativa precaria, con bajos resultados en las pruebas estandarizadas de selección universitaria y menores niveles de capital cultural (Ávalos, 2010; Mizala, Hernández y Makovec, 2011). 
26 FORMACIÓN INICIAL DOCENTE PARA LA EDUCACIÓN INCLUSIVA. ANÁLISIS DE TRES PROGRAMAS CHILENOS DE PEDAGOGÍA EN EDUCACIÓN BÁSICA QUE INCORPORAN LA PERSPECTIVA DE LA EDUCACIÓN INCLUSIVA - C. San Martín, C. Villalobos, C. Muñoz y I. Wyman

En cuanto al segundo aspecto, la evidencia internacional ha destacado que el manejo de la diversidad por parte de los docentes requiere de un periodo formativo extendido y profundo. Así, se ha demostrado el rol central que juega la calidad de las instituciones formadoras (Darling-Hammond \& Baratz-Snowden, 2007) sobre todo considerando que las escuelas más vulnerables son las que concentran más docentes con falencias formativas (Toledo y Valenzuela, 2012). En términos conceptuales, esto es lo que Melnick y Zeichner (1995) han denominado como el "problema de la socialización a través del currículum e instrucción en los programas de Pedagogía" (p. 7). De esta forma, se instala el foco de atención en el proceso formativo al que se enfrentan los aspirantes a docentes, problematizando la centralidad del currículo de los programas y los distintos enfoques e instancias para generar procesos inclusivos. La evidencia comparada en este punto da cuenta de algunas características compartidas por programas eficaces en la inserción de los docentes en aulas altamente diversas, destacándose especialmente tres aspectos:

- la existencia de planes de estudio que enfatizan la importancia del cómo enseñar y la importancia del contexto en la práctica docente;

- el énfasis en la inserción temprana de los estudiantes - a través de la práctica profesional— que incentive el trabajo colaborativo entre docentes novatos y expertos; y

- la generación de trabajos reflexivos sobre la experiencia en aula (Darling-Hammond \& Baratz-Snowden, 2007).

Considerando estos resultados, la evidencia respecto de los planes de estudio de formación docente y su relación con la educación inclusiva ha aumentado notablemente durante la última década, especialmente en Estados Unidos y Europa (Allday, NeilsenGatti \& Hudson, 2013; De Luca, 2012; Florian et al., 2010; Fullerton, Ruben, McBride \& Bert, 2011; Holland, Detgen \& Gutekunst, 2008; Lam y Tung Tsui, 2014). Un estudio acerca de la visión en torno a la diversidad en programas de formación de asistentes de educación en Estados Unidos muestra que el enfoque de los programas analizados ha girado lentamente desde una visión focalizada en las necesidades educativas especiales hacia una visión más integral de la diversidad 
(Pugach \& Blanton, 2012). Por otra parte, estos últimos autores concluyen que los programas analizados por ellos tienen un carácter "transicional" más que "transformacional", es decir, que reflejan la voluntad de abrirse hacia la diversidad, sin ser esta una tarea acabada, lo que se explica por la debilidad en el paso a convertirse en programas que realmente se hagan cargo del desafío de formar para el trabajo en ambientes diversos. Por su parte, Melnick y Zeichner (1995) concluyen que los estudios en torno a la materia indican una clara preferencia por programas con "enfoques de infusión" (infusion approach), caracterizados por integrar la atención por la diversidad a través de todo el programa, por sobre el "enfoque segregado" (segregated approach), el cual trata la diversidad como tema o tópico de varias asignaturas específicas en tanto que otros componentes del programa permanecen inalterables, lo que finalmente, es el dominante entre los programas analizados.

En general, la mayoría de estos estudios comparten dos grandes conclusiones: en primera instancia, la falta de un sentido común entre instituciones formadoras acerca de qué es enseñar en diversidad y generar procesos pedagógicos inclusivos (Darling-Hammond $\&$ Baratz-Snowden, 2007; Pugach \& Blanton, 2012); y en segundo término, la débil articulación entre los diferentes componentes dentro de un mismo programa (malla curricular, descripción, contenidos y objetivos de cursos) (Holland et al., 2008; Melnick \& Zeichner, 1995), que se expresa generalmente en un fuerte énfasis en la presentación de las asignaturas y una presencia más débil de la inclusión en los contenidos que realmente se imparten (Melnick \& Zeichner, 1995), haciendo que en muchas ocasiones estas alusiones nunca llegan a ser más que un marco de contexto dentro de los programas formativos (Pugach \& Blanton, 2012).

En el caso de Chile, no existe mayor evidencia ni estudios que investiguen acerca del enfoque inclusivo de los centros de formación docente en el país desde una perspectiva comparativa, aunque sí existen varias investigaciones de casos particulares de algunas instituciones educativas (Jiménez y Montecinos, 2015; 
28 FORMACIÓN INICIAL DOCENTE PARA LA EDUCACIÓN INCLUSIVA. ANÁLISIS DE TRES PROGRAMAS CHILENOS DE PEDAGOGÍA EN EDUCACIÓN BÁSICA QUE INCORPORAN LA PERSPECTIVA DE LA EDUCACIÓN INCLUSIVA - C. San Martín, C. Villalobos, C. Muñoz y I. Wyman

Tenorio, 2011; Venegas, 2013; Zapata, 2011)5. Por una parte, Jiménez y Montecinos (2015) analizan la relación entre la gestión de la diversidad en aula de profesores novatos y su formación inicial, revelando que existe una brecha entre los aprendizajes entregados y la práctica profesional en relación con la educación inclusiva, ya que los docentes reconocen la falta de herramientas teórico-prácticas para generar cambios pedagógicos y la limitada concepción de la inclusión que se desarrolla en las escuelas. Por otra parte, Tenorio (2011) describe la formación de 42 profesores y profesoras egresadas de una universidad, concluyendo que la educación inclusiva y las necesidades especiales, junto con su abordaje pedagógico, aún no forman parte de una política de la universidad que los considere esenciales en la formación docente.

Otros estudios hacen algunos análisis respecto de casos específicos de programas y cómo se abordan temáticas asociadas con la educación inclusiva, especialmente centrados en la educación multicultural. Zapata (2011) busca conocer la experiencia de formación en diversidad cultural de 80 estudiantes pertenecientes a 10 carreras de Pedagogía de una universidad. El autor concluye que los estudiantes muestran debilidades en cuanto a las herramientas adquiridas para abordar la educación intercultural y que el concepto mismo de educación intercultural no está unificado. En una línea similar, Venegas (2013) indica que en el caso de las universidades pertenecientes al Consejo de Rectores de Chile (CRUCH) solo se observan tres casos en los que se expresa algún tipo de orientación pedagógica hacia la educación inclusiva, que se visibiliza en asignaturas específicas asociadas mayormente con la educación multicultural.

Aunque con distintas metodologías y focos, es posible identificar dos grandes resultados que se desprenden de la evidencia chilena y que, en parte, se vinculan con lo observado en los estudios internacionales. Por un lado, pareciera ser que en ninguno de los casos estudiados se encuentra consolidada y sistematizada la enseñanza de procesos didácticos y pedagógicos asociados con la educación

Asimismo, existen algunas investigaciones que analizan comparativamente la presencia curricular de otros aspectos distintos a la educación inclusiva, como el lenguaje y las matemáticas (Sotomayor et al., 2011; Varas et al., 2008). 
inclusiva (Jiménez y Montecinos, 2015; Tenorio, 2011), dando cuenta de una cierta precariedad en el desarrollo de procesos formativos en torno a la educación inclusiva. Por otra parte, los estudios señalan que la educación inclusiva está fragmentada en distintos enfoques que la integran, pero que no la abordan totalmente, lo que se traduce en la presencia de las distintas temáticas en asignaturas particulares (Venegas, 2013; Zapata, 2011). Corroborar y extender estas hipótesis a través de un estudio comparado de instituciones que incorporan elementos en la educación inclusiva en sus perfiles y descripciones es el objetivo principal de esta investigación.

\section{Metodología}

Para la realización del estudio se utilizó el enfoque de métodos mixtos (Creswell, 2013; Tashakkori y Teddlie, 1998). Los métodos mixtos representan, por definición, un proceso de recolección, análisis e integración de datos provenientes de fuentes cuantitativas y cualitativas, cuyo uso facilita el conocimiento en profundidad de un problema social, entendiendo que las debilidades de un método son, en muchos casos, las fortalezas del otro (Ivankova, Creswell \& Stick, 2006).

\subsection{Diseño de investigación}

El diseño de investigación específico utilizado fue el modelo de "Diseño Explicativo Secuencial" (Sequential Explanatory Design), el cual es una de las estrategias de métodos mixtos más recurrentes en la actualidad (Creswell, 2013). Este método se caracteriza porque la recolección de datos y el análisis de cada una de las fuentes se desarrollan de manera secuencial, para finalmente interpretar los datos de forma conectada. Este diseño es especialmente útil cuando el uso de fuentes cuantitativas y cualitativas busca responder a las mismas interrogantes, pero reconociendo las fortalezas y limitaciones de cada una. Además, tiene a favor su fácil ejecución y simpleza en el seguimiento de los procesos de recolección de datos, análisis e interpretación (Creswell, 2013). Los pasos del diseño se presentan en la Figura 1. 
30 FORMACIÓN INICIAL DOCENTE PARA LA EDUCACIÓN INCLUSIVA. ANÁLISIS DE TRES PROGRAMAS CHILENOS DE PEDAGOGÍA EN EDUCACIÓN BÁSICA QUE INCORPORAN LA PERSPECTIVA DE LA EDUCACIÓN INCLUSIVA - C. San Martín, C. Villalobos, C. Muñoz y I. Wyman

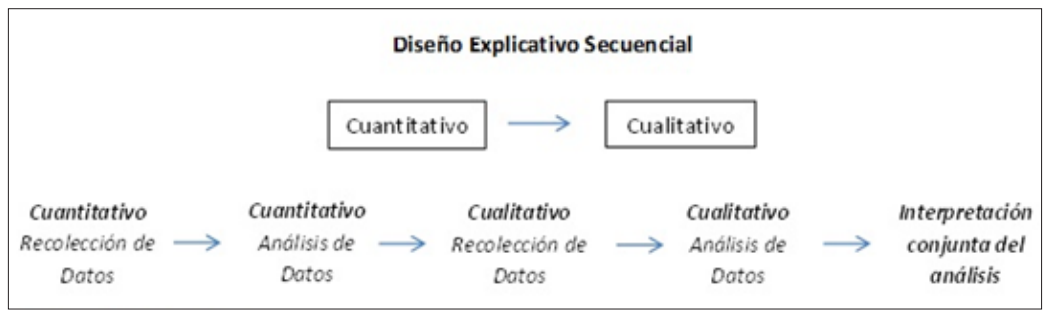

Figura 1. Diseño explicativo secuencial

Fuente: Creswell, 2013, p. 209.

En nuestro caso, el método mixto contempló la utilización de datos cuantitativos y cualitativos. Respecto de los datos cuantitativos, se realizó un análisis de contenido que permitió describir cuantitativamente la frecuencia de conceptos inclusivos en las mallas y perfiles. Por otra parte, el análisis cualitativo se realizó sobre la base de una serie de entrevistas con profesionales involucrados directamente en los programas de Pedagogía.

\subsection{Datos}

La recolección de datos se dividió en dos etapas sucesivas. En la primera, se recolectaron los programas de asignatura que componen la malla curricular (vigente durante el año académico 2015) de tres carreras de Pedagogía en Educación Básica que presentaron una alta alusión a la educación inclusiva en sus perfiles de egreso y descripción de los programas ${ }^{6}$. Dos de los programas escogidos pertenecen a universidades privadas de la Región Metropolitana, mientras que el otro es de una universidad estatal con sede en el norte del país. Para cada programa, se construyó una base de datos que contuvo información básica de cada asignatura: nombre, profesor(es), semestre en que se imparte y tipo, según se tratara de asignaturas teóricas o prácticas. Además, basándonos en Jiménez y Montecinos (2015), se clasificó cada asignatura de acuerdo con el grado en que explícitamente abordan la educación inclusiva, identificando cuatro tipos:

6 Se seleccionaron los cuatro programas que tuvieron 10 o más alusiones a la educación inclusiva. Lamentablemente, los directivos de uno de esos programas declinó participar en el estudio. Para indagar en detalles sobre la selección de los programas con altos niveles de menciones en torno a la educación inclusiva, ver Villalobos, San Martín y Wyman (2016). 
- asignaturas orientadas a la educación inclusiva;

- asignaturas que incorporan parcialmente la educación inclusiva;

- asignaturas que incorporar tangencialmente la educación inclusiva; y

- asignaturas que no incorporan la educación inclusiva ${ }^{7}$.

La unidad de análisis básica fueron los programas de estudio de las asignaturas que componen los tres programas analizados. Mediante un análisis de contenido, se identificaron alusiones a la educación inclusiva a partir del rastreo de conceptos relacionados (Villalobos et al., 2016).

En una segunda etapa, se realizaron entrevistas semiestructuradas (Taylor y Bogdan, 1987) a seis personas, incluyendo directores de los programas y a docentes de las asignaturas orientadas a la educación inclusiva, con el fin de conocer en mayor profundidad cómo los actores involucrados en la formación docente la entienden, la importancia que le otorgan dentro del plan formativo, así como también, la existencia de otras instancias que, por el contrario, podrían formar parte del currículo oculto (Jackson, 1991) de la formación de profesores de educación básica.

\subsection{Análisis}

A partir de la base de datos que contenía la información de las mallas curriculares y programas de asignaturas, se realizó un análisis descriptivo que permitió identificar qué tipos de asignatura — de

\footnotetext{
La definición de cada categoría es la siguiente: a) Asignaturas orientadas a la educación inclusiva: Su temática y objetivo formativo central se relaciona con la perspectiva y discusión sobre la educación inclusiva, lo que se manifiesta en el nombre del curso, su descripción, las competencias o habilidades que busca desarrollar y/o objetivo principal del curso; b) Asignaturas que incorporan parcialmente elementos de educación inclusiva: La educación inclusiva forma parte de alguno de los objetivos específicos de la asignatura, sin ser esta la temática central. Esto se manifiesta en que los ámbitos o discusiones acerca de la educación inclusiva están presentes en la asignatura, pero tienen un lugar secundario; c) Asignaturas que incorporan tangencialmente elementos de educación inclusiva: Incluyen sugerencias indirectas a la educación inclusiva, generalmente limitadas a la contribución de estándares y competencias genéricas del perfil de egreso, sin tener componentes, dimensiones o áreas directamente vinculadas con la perspectiva de la educación inclusiva; d) Asignaturas que no incorporan elementos de educación inclusiva: No incluyen ni directa ni indirectamente elementos de educación inclusiva en sus programas de asignatura.
} 
32 FORMACIÓN INICIAL DOCENTE PARA LA EDUCACIÓN INCLUSIVA. ANÁLISIS DE TRES PROGRAMAS CHILENOS DE PEDAGOGÍA EN EDUCACIÓN BÁSICA QUE INCORPORAN LA PERSPECTIVA DE LA EDUCACIÓN INCLUSIVA - C. San Martín, C. Villalobos, C. Muñoz y I. Wyman

acuerdo con su orientación a la educación inclusiva - caracterizan a cada programa, dónde se ubican las asignaturas con mayor énfasis en esta materia y finalmente, qué los caracteriza de acuerdo con la manera en que abordan la inclusión. Por su parte, con las entrevistas hechas y transcritas, se utilizó la técnica de análisis del contenido (Krippendorff, 1990; Lincoln \& Denzin, 1994) y se realizó una codificación abierta (Andreu, 2002) de las principales categorías. Finalmente, se triangularon los resultados de los análisis obtenidos previamente para efectuar interpretaciones finales, basándose en el modelo de Creswell (2013), donde las entrevistas complementan la información obtenida a partir de los datos cuantitativos, lo que permite considerar si la información responde un análisis de acuerdo/ convergencia, complementariedad, discrepancia o disonancia (Farmer, Robinson, Elliott \& Eyles, 2006).

\section{Resultados}

La presentación de los resultados se estructura en tres apartados, abarcando las preguntas centrales del estudio: ¿de qué forma se incorpora la educación inclusiva en la formación inicial de docentes de educación básica?, ¿en qué momento de los programas se incorporan los elementos de la educación inclusiva? y, ¿bajo qué conceptos se entiende la idea de la educación inclusiva? La evidencia proporcionada por ambas fuentes de información se presenta de manera conectada (Creswell, 2013).

\subsection{Incorporación de la educación inclusiva en los programas}

En primer lugar, a partir del análisis descriptivo de los tipos de asignatura, se buscó dar cuenta de la importancia que reviste la educación inclusiva en los programas de formación inicial docente para los académicos formadores y representantes de cada uno de los programas analizados. En la Tabla 1 se presenta la frecuencia de asignaturas según el grado de compromiso de cada una respecto de la promoción de la educación inclusiva. 
Tabla 1

Distribución de cursos según nivel de abordaje de la educación inclusiva

\begin{tabular}{lcccccc}
\hline & \multicolumn{2}{c}{ Universidad 1 } & \multicolumn{2}{c}{ Universidad 2 } & \multicolumn{2}{c}{ Universidad 3 } \\
\hline & $N$ & $\%$ & $N$ & $\%$ & $N$ & $\%$ \\
Totalmente orientado & 4 & $9,30 \%$ & 2 & $8,33 \%$ & 3 & $5,66 \%$ \\
Parcialmente orientado & 12 & $27,91 \%$ & 6 & $25,00 \%$ & 17 & $32,08 \%$ \\
Tangencialmente orientado & 15 & $34,88 \%$ & 12 & $50,00 \%$ & 24 & $45,28 \%$ \\
No orientado & 12 & $27,91 \%$ & 4 & $16,67 \%$ & 9 & $16,98 \%$ \\
Total & 43 & $100 \%$ & $24 *$ & $100 \%$ & 53 & $100 \%$ \\
\hline
\end{tabular}

$N=$ Número de cursos.

* Los cursos de la Universidad 2 son anuales.

Fuente: Elaboración propia.

Los datos muestran que el compromiso de estos programas está caracterizado por una orientación más bien secundaria o "transicional" hacia la inclusión, que se manifiesta en la predominancia de las asignaturas que tienen una orientación tangencial y parcial hacia esta temática, siendo bajo el número de asignaturas que abordan directamente la materia, especialmente en un caso (Universidad 2). Este patrón es común en los tres programas y sugiere que la formación en torno a la educación inclusiva se verifica de un modo más implícito que explícito, a lo menos en lo que refiere a los programas de estudio. Adicionalmente, en todos los programas más del 15\% de las asignaturas no incorpora ningún concepto relativo a la educación inclusiva, lo que llega a más de un $25 \%$ en un caso (Universidad 1), revelando así la existencia de un grupo relevante de asignaturas (y de profesores) que no ha incorporado en ningún aspecto la perspectiva de la educación inclusiva.

La información obtenida a partir de las entrevistas corrobora, en general, estos datos. Así, el discurso de los directores de carrera apunta hacia la necesidad de hacer transversales los contenidos asociados a la educación inclusiva en las distintas asignaturas, lo que se visualiza como una tarea aún pendiente. De esta manera, los directivos revelan que durante los últimos años se han hecho modificaciones en las asignaturas para lograr que las competencias relacionadas con la educación inclusiva adquieran un carácter más 
34 FORMACIÓN INICIAL DOCENTE PARA LA EDUCACIÓN INCLUSIVA. ANÁLISIS DE TRES PROGRAMAS CHILENOS DE PEDAGOGÍA EN EDUCACIÓN BÁSICA QUE INCORPORAN LA PERSPECTIVA DE LA EDUCACIÓN INCLUSIVA - C. San Martín, C. Villalobos, C. Muñoz y I. Wyman

transversal, superando la particularidad de las asignaturas, aunque esto no siempre se ha cumplido como se ha esperado, tal como indica un directivo:

Entrevistado: Lo que pasa es que el programa "X" era un programa que funcionaba nucleado, ise entiende?, pero el programa "Y" rompe este núcleo, porque no sé, era Ciencias Naturales con Ciencias Sociales e iban en un núcleo, entonces los profesores tenían que evaluar juntos, un proyecto integral, súper lindo, pero no dio los resultados del Inicia ${ }^{8}$. Esa fue la razón, y era anual, ahora es un sistema semestral, más clásico, y ya esa asignatura no continuó en el programa.

Entrevistador: Pero en el fondo ¿o la remplazaron por otra que fuera una asignatura o lo están viendo de manera más transversal? Entrevistado: No, más transversal, claro, mira hay orientación y liderazgo, por ejemplo, se trabaja mucho el tema de la inclusión (Director Universidad 2).

Relacionado con lo anterior, se visualiza un esfuerzo por parte de los responsables de los programas por potenciar los procesos para hacer transversales los componentes de la educación inclusiva en las distintas asignaturas, para lo que se requiere pasar de acciones discursivas a acciones prácticas que transformen los contenidos del conjunto de los programas, tal como destaca otro directivo:

Las prácticas pedagógicas, yo creo que tenemos declaraciones instaladas en los programas, en algunos programas encuentro muy importante lo que tú estás diciendo, no sé si agregarle la competencia de "valor a la inclusión y a la diversidad" y "aprender" a todos los programas sea la solución, yo no creo que vaya por ahí, creo que va más en formarnos como docentes en competencias que tenemos que nosotros desarrollar para instalar en nuestras aulas y nuestra esencia y modelar el tema, claramente algunas asignaturas tienen que tener la declaración, porque cae de cajón, o sea son más pertinentes, pero cuando yo haga, no sé, la asignatura de datos y azar es químicamente puro, si podríamos unir el

Programa para la formación inicial docente. 
tema de la inclusión igual, y de hecho está, ¿te fijas? (Directora Universidad 1).

Es importante mencionar que los entrevistados concuerdan con la necesidad de sistematizar las estrategias llevadas a cabo por los académicos en cada aula para potenciar y desarrollar la perspectiva de la educación inclusiva. Partiendo del supuesto de que la mayoría de los docentes posee conocimientos y conceptos diferentes, la generación de estrategias de integración entre docentes y asignaturas emerge como un tema central para la transversalización de la educación inclusiva, tal como se revela en la siguiente cita:

Yo creo que de hecho intenté hacer acciones, pero no llegan a puerto y tiene que ver con los tiempos de uno y uno se siente súper culpable, por ejemplo esto de identificación de necesidades educativas, que es en la práctica, siempre hemos hablado, nos tenemos que reunir con las profes de práctica de básica, porque para mí es esencial que también no vean que es una tarea aparte, una pega aparte y que los chiquillos tampoco los tenían así, que un minuto los sintieron el año pasado, por eso también lo analizo, pero eso no se ha dado, o creo que definitivamente el programa en sí debería ser sumamente compartido principalmente con los profes de práctica, digo principalmente no sé si todos, pero sí los profes de práctica, entonces en un minuto creo que se está dando todavía desde una perspectiva un poquito aislada, todavía siguen su mundo (Académica, Universidad 1).

Finalmente, es relevante considerar que en un intento de transversalizar las temáticas referidas a inclusión y pedagogía para la diversidad, los entrevistados se refieren a sus esfuerzos por potenciar el desarrollo de competencias para la colaboración profesional como un aspecto clave para la coenseñanza educativa. En este sentido, los directores de las Universidades 1 y 2 manifestaron la existencia de un vínculo intencionado con académicos y estudiantes de las carreras de educación diferencial. Sin embargo, estas relaciones parecen aún acotadas a algunas asignaturas, sin existir procesos sinérgicos de largo plazo entre ambos grupos de académicos y estudiantes, tal como indica un directivo: 
36 FORMACIÓN INICIAL DOCENTE PARA LA EDUCACIÓN INCLUSIVA. ANÁLISIS DE TRES PROGRAMAS CHILENOS DE PEDAGOGÍA EN EDUCACIÓN BÁSICA QUE INCORPORAN LA PERSPECTIVA DE LA EDUCACIÓN INCLUSIVA - C. San Martín, C. Villalobos, C. Muñoz y I. Wyman

Por otro lado, otra cosa que a mí me ha pasado, que lo hemos conversado mucho, que es un súper desafío que es: ok, nosotros tenemos esta declaración y esta forma de gestionar el currículum en que los chiquillos de básica y de diferencial estudian juntos Lenguaje y Matemáticas, pero yo creo que nos falta algo todavía, en lograr ahí una inclusión y una integración entre ellos; de verdad lo hemos tenido que trabajar, con los profesores, los profesores tienden a hacer el discurso de Básica, y les cuesta y los mismos chiquillos de Diferencial han reclamado las clases a ser más inclusivos, entonces se ha hecho un trabajo, el equipo de la directora de la carrera ha hecho un trabajo con los profesores, pero yo creo que todavía tenemos mucho que avanzar, mucho, mucho, ya estamos haciendo una innovación que yo entiendo que nadie más en Chile está haciendo, pero por lo mismo lo hemos pensado harto con la directora de la carrera: cómo hacer un proceso que nos permita hacer esta apuesta, sistematizarla y consolidarla (Directora, Universidad 1).

\subsection{Ubicación de las asignaturas de educación inclusiva en los programas}

Complementando estos resultados, el análisis de la ubicación temporal de las asignaturas permite relevar la intención de las facultades de dar coherencia a la secuencia del currículo, si es que se encuentran relacionados de alguna manera y/o si se asegura que las asignaturas lo hagan a lo largo de todo el programa (Blanton \& Pugach, 2011). En el caso de los programas estudiados, la Figura 2 presenta el porcentaje de asignaturas (en relación con el total) que tienen algún grado de orientación (total o parcial) hacia la educación inclusiva. Los resultados indican que los componentes de la educación inclusiva se dan, en todos los casos, a lo largo de toda la formación inicial docente, aunque con menor intensidad en el inicio y final de la carrera. 


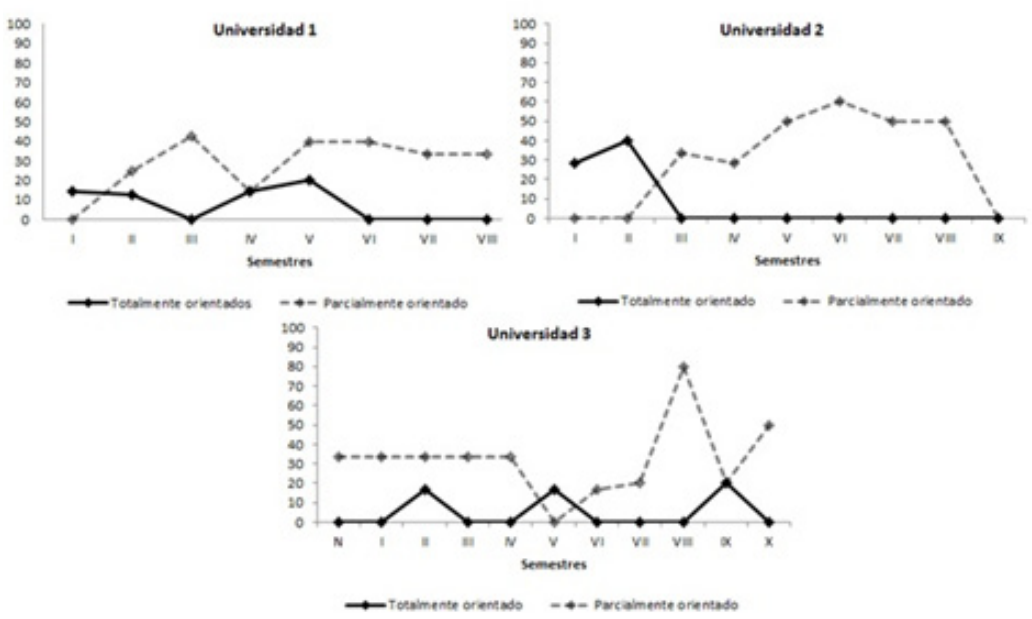

Figura 2. Ubicación temporal de cursos con orientación total o parcial hacia la educación.

Fuente: Elaboración propia.

Si bien estos resultados complementan lo expresado anteriormente acerca de la necesidad (pendiente) de transversalizar los contenidos asociados a la educación inclusiva, la ubicación temporal de estas asignaturas no fue una temática que emergiera con fuerza en el discurso de los actores clave. Solo una de las directoras de los programas reveló la importancia de la ubicación de las asignaturas, en tanto que estas se desarrollan a lo largo de todo el plan curricular, enfatizando en los beneficios que puede traer impulsar desde el primer año el enfoque inclusivo.

Bueno, es que ahí donde yo creo, bueno de partida la asignatura de Didácticas Generales nosotros lo eliminamos del plan 4, lo que hicimos fue que como te digo, hicimos Educación en Derechos Humanos y Diversidad, el tema de convivencia lo pasamos a una asignatura que se llama Profesor Jefe y Convivencia en la Escuela; el tema de ciudadanía lo instalamos — que también tiene mucho que ver con el tema de inclusión-, lo instalamos dentro de una asignatura de Ciencias Sociales, de Estudios Sociales, y potenciamos más la asignatura de Necesidades Educativas Especiales y lo trasladamos al segundo semestre de la carrera, esa 
38 FORMACIÓN INICIAL DOCENTE PARA LA EDUCACIÓN INCLUSIVA. ANÁLISIS DE TRES PROGRAMAS CHILENOS DE PEDAGOGÍA EN EDUCACIÓN BÁSICA QUE INCORPORAN LA PERSPECTIVA DE LA EDUCACIÓN INCLUSIVA - C. San Martín, C. Villalobos, C. Muñoz y I. Wyman

es otra decisión importante que tomamos, o sea antes la secuencia era esta: mira, fíjate, en segundo año partían con Currículum y Evaluación y en tercer año tenían Necesidades Educativas Especiales; nosotros invertimos la cosa, en primer semestre tienen Currículum, donde nosotros ya instalamos el tema de la contextualización y parte de lo que tiene que ir a ver en la práctica es qué pasa con la diversidad y forma de aprendizaje en los niños y en el segundo semestre tiene Psicología del Aprendizaje junto con Necesidades Educativas Especiales, o sea adelantamos dos años y van también a la escuela también a mirar cómo hacen adecuaciones curriculares y cómo las áreas del aprendizaje me iluminan esta necesidad y formas de aprender de los niños, esa fue otra decisión que tomamos y Evaluación lo pasamos a tercer año, es decir, los estudiantes en el primer año le damos el constructo así full, y le decimos, "mire, de aquí en adelante nunca más puede dejar de mirar desde esta forma" (Directora Universidad 1).

\subsection{Conceptualización de la educación inclusiva en la formación inicial docente}

Para responder a la pregunta respecto de cómo entienden los actores formadores la educación inclusiva en un contexto de formación de profesores, se analizó la frecuencia de menciones de conceptos alusivos a la educación inclusiva en los tres programas de PEB mencionados. La Tabla 2 muestra el número de menciones y porcentaje que representa cada una dentro del programa, según los distintos conceptos de educación inclusiva revisados. 
Tabla 2

Frecuencia de menciones a conceptos alusivos a la educación inclusiva

\begin{tabular}{|c|c|c|c|c|c|c|c|c|c|}
\hline \multirow[t]{2}{*}{ Dimensión } & \multirow[t]{2}{*}{ Concepto } & \multicolumn{2}{|c|}{ Universidad 1} & \multicolumn{2}{|c|}{ Universidad 2} & \multicolumn{2}{|c|}{ Universidad 3} & \multicolumn{2}{|c|}{ Total } \\
\hline & & $N$ & $\%$ & $N$ & $\%$ & $N$ & $\%$ & $N$ & $\%$ \\
\hline \multirow{5}{*}{$\begin{array}{l}\text { Principios } \\
\text { y valores } \\
\text { declarados }\end{array}$} & Diversidad & 15 & 17,4 & 19 & 22,9 & 6 & 7,1 & 40 & 15,7 \\
\hline & Inclusión & 6 & 7 & 15 & 18,1 & 3 & 3,5 & 24 & 9,4 \\
\hline & Democracia & 4 & 4,7 & 2 & 2,4 & 1 & 1,2 & 7 & 2,8 \\
\hline & Derechos Humanos & 3 & 3,5 & 1 & 1,2 & 0 & 0 & 4 & 1,6 \\
\hline & Otros & 7 & 8,1 & 2 & 2,4 & 6 & 7,1 & 15 & 5,9 \\
\hline \multirow{5}{*}{$\begin{array}{l}\text { Componentes } \\
\text { de la } \\
\text { diversidad }\end{array}$} & Consideración del contexto & 14 & 16,3 & 19 & 22,9 & 6 & 7,1 & 39 & 15,4 \\
\hline & Consideración étnica & 2 & 2,3 & 0 & 0 & 28 & 32,9 & 30 & 11,8 \\
\hline & $\begin{array}{l}\text { Consideración de todos los } \\
\text { estudiantes }\end{array}$ & 8 & 9,3 & 5 & 6 & 17 & 20 & 30 & 11,8 \\
\hline & Consideración de NEE & 3 & 3,5 & 2 & 2,4 & 2 & 2,4 & 7 & 2,8 \\
\hline & Consideración de género & 3 & 3,5 & 0 & 0 & 0 & 0 & 3 & 1,2 \\
\hline \multirow{3}{*}{$\begin{array}{l}\text { Gestión } \\
\text { curricular } \\
\text { para la } \\
\text { inclusión }\end{array}$} & Enseñanza adaptativa & 19 & 22,1 & 7 & 8,4 & 9 & 10,6 & 35 & 13,8 \\
\hline & Colaboración profesional & 2 & 2,3 & 9 & 10,8 & 6 & 7,1 & 17 & 6,7 \\
\hline & $\begin{array}{l}\text { Colaboración familia- } \\
\text { comunidad }\end{array}$ & 0 & 0 & 2 & 2,4 & 1 & 1,2 & 3 & 1,2 \\
\hline
\end{tabular}

$N$ = número de menciones.

Fuente: Elaboración propia.

Como se observa en la Tabla 2, entre los valores asociados a la educación inclusiva indicados en los programas de asignatura priman claramente la diversidad y la inclusión, ambos conceptos son recurrentes en los tres programas. Por su parte, como componentes esenciales de la enseñanza inclusiva existe un alto nivel de menciones referidas al contexto en el que se lleva a cabo la enseñanza y a la consideración de todos los estudiantes. En el programa de la Universidad 3, dada su orientación multicultural, prevalece también la atención a la inclusión y hacia las diferencias étnicas, lo que podría tener relación con los estudiantes, así como con la manera de llevar a cabo procesos pedagógicos eficaces en contextos de alta diversidad. Entre los elementos asociados al trabajo curricular en favor de la educación inclusiva, se destaca la importancia de generar procesos de enseñanza adaptativos y, en menor medida, la importancia de la colaboración entre profesionales.

Cuando se compara la prevalencia de estos conceptos entre los programas de asignatura, el perfil de egreso y la malla curricular se puede observar que no existe un correlato entre ambos instrumentos 
40 FORMACIÓN INICIAL DOCENTE PARA LA EDUCACIÓN INCLUSIVA. ANÁLISIS DE TRES PROGRAMAS CHILENOS DE PEDAGOGÍA EN EDUCACIÓN BÁSICA QUE INCORPORAN LA PERSPECTIVA DE LA EDUCACIÓN INCLUSIVA - C. San Martín, C. Villalobos, C. Muñoz y I. Wyman

(ver Tabla 3). En este sentido, existen elementos presentes en el perfil de egreso que no forman parte ni directa ni indirectamente de los objetivos o contenidos de las asignaturas del programa, dando cuenta así de un desacople entre ambos instrumentos curriculares.

Tabla 3

Comparación de la presencia de conceptos alusivos a la educación inclusiva en perfil de egreso y malla curricular

\begin{tabular}{|c|c|c|c|c|c|c|c|}
\hline \multirow[t]{2}{*}{ Dimensión } & \multirow[t]{2}{*}{ Concepto } & \multicolumn{2}{|c|}{ Universidad 1} & \multicolumn{2}{|c|}{ Universidad 2} & \multicolumn{2}{|c|}{ Universidad 3} \\
\hline & & $\mathrm{PE}$ & $M C$ & $\mathrm{PE}$ & MC & $P E$ & $M C$ \\
\hline \multirow{5}{*}{$\begin{array}{l}\text { Principios } \\
\text { y valores } \\
\text { declarados }\end{array}$} & Diversidad & 7,1 & 17,4 & 13,3 & 22,9 & 22,2 & 7,1 \\
\hline & Inclusión & 14,3 & 7 & 10 & 18,1 & 0 & 3,5 \\
\hline & Democracia & 7,1 & 4,7 & 3,3 & 2,4 & 0 & 1,2 \\
\hline & Derechos humanos & 14,3 & 3,5 & 0 & 1,2 & 5,6 & 0 \\
\hline & Otros & 21,4 & 8,1 & 20 & 2,4 & 27,8 & 7,1 \\
\hline \multirow{5}{*}{$\begin{array}{l}\text { Componentes } \\
\text { de la diversidad }\end{array}$} & Consideración del contexto & 0 & 16,3 & 6,7 & 22,9 & 0 & 7,1 \\
\hline & Consideración étnica & 0 & 2,3 & 0 & 0 & 33,3 & 32,9 \\
\hline & Consideración de todos los estudiantes & 7,1 & 9,3 & 6,7 & 6 & 0 & 20 \\
\hline & Consideración de NEE & 0 & 3,5 & 10 & 2,4 & 0 & 2,4 \\
\hline & Consideración de género & 7,1 & 3,5 & 10 & 0 & 0 & 0 \\
\hline \multirow{3}{*}{$\begin{array}{l}\text { Gestión } \\
\text { curricular para } \\
\text { la inclusión }\end{array}$} & Enseñanza adaptativa & 0 & 22,1 & 10 & 8,4 & 11,1 & 10,6 \\
\hline & Colaboración profesional & 7,1 & 2,3 & 10 & 10,8 & 0 & 7,1 \\
\hline & Colaboración familia-comunidad & 14,3 & 0 & 0 & 2,4 & 0 & 1,2 \\
\hline
\end{tabular}

Nota: Los datos de la tabla corresponden a porcentajes

$\mathrm{PE}=$ Perfil de egreso; $\mathrm{MC}=$ Malla curricular

Fuente: Elaboración propia.

A partir de las entrevistas, es posible reconocer que, en general, el discurso de los entrevistados tiene un enfoque amplio respecto de cómo entienden la educación inclusiva y que en parte confirma los resultados cuantitativos, aun cuando se evidencian contradicciones. Así, tanto la mayoría de directores como de académicos asocia la educación inclusiva con el valor de la diversidad, convergiendo en la idea de que esta no puede entenderse solo desde un enfoque asociado a las necesidades especiales o las diferencias culturales, sino que debe superar esta visión e integrar la multiplicidad de formas de aprendizaje, tal como se visualiza en la siguiente idea:

[...] o sea, para mí la inclusión y diversidad no están solo enfocadas en cómo trabajar con niños de capacidades educativas especiales permanentes en lo que es la tradición del diagnóstico 
y encasillar a los niños, sino que la diversidad e inclusión tienen que ver con reconocer las características que tiene el estudiante, profundizar en la contextualización de mi aula y sobre eso tomar decisiones pedagógicas que estén centradas en que todos puedan realmente aprender, todos y todas (Directora, Universidad 1).

De esta forma, es posible observar cómo algunos de los componentes de la diversidad son integrados en los discursos, especialmente asociados con la consideración del contexto y de los estudiantes. Sin embargo, en uno de los casos se pone especial énfasis en el tema de la consideración étnica, dado que el foco de la carrera es la educación intercultural. Quizás por esto, la directora no aborda la inclusión como tal ni como un enfoque principal de la carrera, sino que se alinea con una visión de la educación que es dialógica respecto del mundo indígena:

Lo que nosotros pretendíamos era que esta malla no fuera inclusiva, sino que fuera, o sea esta malla no va a incluir lo indígena como una cosa así "ya, lo vamos a considerar", sino que el ejercicio es distinto, nosotros pensamos que el mundo indígena es un mundo que históricamente, a partir de que nuestro país genera el concepto de Estado, comienza a ver que es la comunidad o son comunidades, siempre, y eso ha sido por un proceso histórico y todo lo que queramos saber de ellos y todo lo que el mundo sabe por un tema de trayectoria, es un tema fronterizo, un tema de diferenciación (...), pero lo que nosotros pensamos al día de hoy es que nuestra malla es distinta, porque nuestra malla lo que pretende en ese ejercicio de buscar este diálogo que se habla mucho del diálogo que hay que tener un diálogo, el diálogo se plantea siempre en simetría, cuando hablo con un otro estamos hablando en las mismas condiciones, ¿cierto? (...) entonces lo que promueve esta malla es fortalecer el proceso indígena, de la identidad, de la revaloración, del redescubrimiento de lo propio en los chiquillos (Directora, Universidad 3).

Adicionalmente, las entrevistas revelaron que, si bien los académicos y directores tienen una definición relativamente similar de la idea de la educación inclusiva, no hay claridad de que esta visión 
42 FORMACIÓN INICIAL DOCENTE PARA LA EDUCACIÓN INCLUSIVA. ANÁLISIS DE TRES PROGRAMAS CHILENOS DE PEDAGOGÍA EN EDUCACIÓN BÁSICA QUE INCORPORAN LA PERSPECTIVA DE LA EDUCACIÓN INCLUSIVA - C. San Martín, C. Villalobos, C. Muñoz y I. Wyman

también sea compartida por los estudiantes. Esto se debería, en parte, a que en las políticas educativas (y en el discurso instalado) se sigue vinculando el concepto de educación inclusiva con las necesidades educativas especiales de los potenciales alumnos, tal como se refleja en la siguiente cita:

Yo creo que todavía estamos pobres, o sea, estos cabros yo creo que les cuesta todavía hacer una planificación con adaptación curricular, les cuesta visualizar una metodología distinta para grupos distintos más bien, van con una clásica y yo creo que ellos tiene como en su mente que la inclusión o la diversidad la van a trabajar personalmente, o sea me voy acercar al chiquillo y yo lo voy ayudar ahí, y no en una metodología que integre todo esto, ¿te das cuenta? Yo creo que todavía está el patrón de que la diferencia la atiendo personalmente digamos, un apoyo directo (Director, Universidad 2).

Además, los directores reconocen la falta de procesos de monitoreo de lo que se enseña en las salas de clases a los futuros docentes, dando cuenta de una limitación organizacional fundamental para poder desarrollar de manera más potente la perspectiva de la educación inclusiva, tal como se muestra en las siguientes citas de dos directivos de las universidades analizadas:

Hoy día estamos trabajando en un proceso intermedio de ir monitoreando año a año. Ya, pero este diseño en que los profes participan uno sabe cuán comprometidos están en este diseño, y aquí vienen sus propias culturas, sus casas formadoras, todo, o sea aquí viene un profe nuevo, entraron tres profes nuevos, son tres profes que traen escuela de origen distinto, que traen cultura, que traen creencias, que traen expectativas y la escuela que además tiene su cultura, sus creencias, sus expectativas, su manera de mirar el mundo, etc. y estas dos culturas se van a confrontar siempre y de esto va a salir un híbrido para ver donde tendió esto... es muy difícil, entonces ahí estamos encima, estamos monitoreando, estamos reorientando, estamos trabajando en un plan de estudio nuevo, pero seguimos en el plano de una gran apuesta, esa es la palabra (Director, Universidad 2). 
Van apareciendo profesores y esos profesores no necesariamente son profesores que han estado en conocimiento de la experiencia del programa. Lo que nosotros solemos hacer siempre -y siempre lo hacemos con ellos o sin ellos- es tener muchas reuniones, y eso es como lo más agotador, porque significa romper un esquema mental del otro, a veces cuesta mucho porque hay resistencias que son muy inconscientes las resistencias, pero siempre te quieren plantear un discurso como si esto otro ya se ha hecho de esta otra manera y da resultado, y yo le digo, estamos trabajando con este otro plan que es un plan integrador, un plan distinto, que hay que trabajar distinto. Aquí una asignatura la hacen dos tres personas, no va una sola, entonces como yo converso con ellos, como vamos evidenciando eso que se note en los resultados y esos productos, ellos tiene que mostrar productos, entonces esto no solamente es importante decir "lo hicimos": ellos tiene que mostrar que fueron capaces de crear materiales interculturales, y eso puede ser en lengua, o usar en matemática, te fijas, que ellos sean capaces de ofrecer una planificación integradora y que puedan ser capaces de entrar (Director, Universidad 3).

\section{Conclusiones}

El estudio analizó la forma en que tres de los programas de Pedagogía en Educación Básica en Chile, que incorporan elementos de la educación inclusiva en sus descripciones y perfiles de egreso, incluían dentro de su currículo formativo un enfoque educativo inclusivo. En sintonía con otros estudios internacionales (Holland et al., 2008; Melnick \& Zeichner, 1995; Pugach \& Blanton, 2012) los resultados mostraron que:

- la mayoría de los programas incorpora temáticas referidas a educación inclusiva en asignaturas específicas, por lo que se presentan desafíos para transversalizar estos elementos en el proceso de la FID $^{9}$;

\footnotetext{
Si bien muchas de las asignaturas son disciplinarias, consideramos que la transversalización de la perspectiva de la educación inclusiva permite dar coherencia y profundidad a la formación docente en esta materia, en línea con lo indicado por diversos estudios internacionales (Melnick \& Zeichner, 1995; Pugach \& Blanton, 2012; De Luca, 2012).
} 
44 FORMACIÓN INICIAL DOCENTE PARA LA EDUCACIÓN INCLUSIVA. ANÁLISIS DE TRES PROGRAMAS CHILENOS DE PEDAGOGÍA EN EDUCACIÓN BÁSICA QUE INCORPORAN LA PERSPECTIVA DE LA EDUCACIÓN INCLUSIVA - C. San Martín, C. Villalobos, C. Muñoz y I. Wyman

- los programas de formación inicial docente analizados presentan importantes diferencias entre sus distintos componentes (perfiles de egreso, mallas curriculares y discursos institucionales), lo que implica que, en general, no existen altos niveles de coherencia interna respecto de cómo se desarrolla la educación inclusiva en los distintos programas; $y$

- la mayoría de los programas desarrolla una aproximación hacia la educación inclusiva basada en el discurso, más que en la implementación de prácticas pedagógicas concretas, lo que se observa tanto en el análisis de los componentes (donde no hay un mayor énfasis en el trabajo referido a la gestión curricular) como en el discurso de los entrevistados (que es bastante amplio e inespecífico).

Desde nuestra perspectiva, esta evidencia muestra un conjunto de tensiones existentes entre el sistema escolar y el sistema de formación docente. La reciente promulgación de una serie de iniciativas gubernamentales orientadas a aumentar la mixtura y diversidad en el sistema escolar (por ejemplo, Ley No 20.845 y Decreto Exento 83/2015) ha incrementado la presión hacia los docentes respecto de la incorporación de perspectivas, competencias y conocimientos relacionados con la educación inclusiva. Esta demanda, sin embargo, no ha encontrado necesariamente como correlato una transformación estructural de los programas de Pedagogía, los que debido a su alto grado de autonomía, su orientación al mercado y su clivaje organizacional universitario, han respondido solo gradualmente a la demanda por mayores niveles de inclusión en el ámbito educativo. Esta situación podría ser una de las razones por la cual los profesores manifiesten no sentirse capacitados para responder educativamente a todos sus estudiantes (Damm, 2009; Jordan, Schwartz \& McGhieRichmond, 2009) y que la educación de estudiantes con características particulares sea aún concebida como responsabilidad de profesores especialistas (Florian, 2012; Infante, 2010).

Este escenario promueve, por lo tanto, una serie de desafíos para la formación inicial docente en el país, entre los que pueden destacarse especialmente cuatro. Por una parte, los resultados demuestran (y en parte, confirman) la necesidad de que las Escuelas 
de Pedagogía desarrollen marcos comunes de acción, orientados o promovidos desde el Estado, que puedan generar las condiciones para que todos los futuros profesores cuenten con mayores oportunidades de aprendizaje para desarrollar competencias que les permitan planificar, implementar y evaluar sus prácticas pedagógicas, considerando la diversidad de todos sus estudiantes y asumiendo que las diferencias son un aspecto esencial e inherente del desarrollo y aprendizaje humano (Booth \& Ainscow, 2011; Florian, 2007).

En segundo término, parece fundamental que los programas de formación inicial docente profundicen en el desarrollo de un enfoque integrador, centrado en la colaboración y diálogo entre los planes de formación de profesores de enseñanza regular y especial, potenciando el desarrollo y aprendizaje de estrategias para trabajar colaborativamente con otros (Florian, 2007). Esto permitiría abandonar la lógica de división del trabajo educativo existente en el país, potenciando el trabajo colaborativo entre los distintos profesionales de la educación que se desempeñan al interior de los establecimientos, en pos del desarrollo de una perspectiva común de la educación inclusiva.

Un tercer desafío refiere a los enfoques pedagógicos de la FID para la educación inclusiva, específicamente respecto del desarrollo de estos conocimientos y habilidades en asignaturas específicas o desde enfoques "integrados". Aun cuando hay un número limitado de investigaciones acerca de modelos de formación de profesores para la inclusión, de modo de asegurar la efectividad de uno u otro modelo (Forlin, 2012), es fundamental que independientemente del enfoque que se adopte, el énfasis de la formación inicial docente se centre en el desarrollo de asignaturas que impacten en la forma de pensar y actuar de los futuros profesores hacia la construcción de una escuela para todos (European Agency for Development in Special Needs Education, 2011).

Finalmente, y relacionado con lo anterior, parece central ampliar el concepto mismo de inclusión, involucrando de manera activa procesos reflexivos que contribuyan al desarrollo y transformación de actitudes, creencias y valores coherentes y 
46 FORMACIÓN INICIAL DOCENTE PARA LA EDUCACIÓN INCLUSIVA. ANÁLISIS DE TRES PROGRAMAS CHILENOS DE PEDAGOGÍA EN EDUCACIÓN BÁSICA QUE INCORPORAN LA PERSPECTIVA DE LA EDUCACIÓN INCLUSIVA - C. San Martín, C. Villalobos, C. Muñoz y I. Wyman

necesarios para el desarrollo de una educación inclusiva. Además, es fundamental que los programas de formación inicial de todo el profesorado promuevan el desarrollo de habilidades y conocimientos que potencien la gestión curricular inclusiva, entregando así a los futuros profesores herramientas para diversificar sus prácticas pedagógicas (Hardam, 2009) y potenciar el desarrollo de escuelas con culturas, políticas y prácticas inclusivas (Booth \& Ainscow, 2011).

Junto con estas perspectivas de política pública y orientaciones para la formación inicial docente, los resultados de este estudio pueden considerarse como un insumo relevante para el desarrollo y profundización de nuevas investigaciones en torno a la misma y al sistema escolar en la temática de la educación inclusiva. Por una parte, parece interesante indagar en la forma en que los aprendizajes de la educación inclusiva persisten (o no) luego de que los estudiantes egresan. Estudios preliminares (Jiménez y Montecinos, 2015) muestran la existencia de un fuerte desacople entre la perspectiva de una institución de educación superior y el discurso de sus egresados acerca de la educación inclusiva multicultural, pero no entregan mayores datos comparativos para analizar la estabilidad de esta tendencia en otros espacios educativos. Por otro lado, sería importante profundizar en otros espacios de implementación del currículo y de los programas de Pedagogía (como la bibliografía entregada, las evaluaciones utilizadas o los documentos oficiales diseñados) para evaluar en qué medida el desacople evidenciado entre el perfil de egreso, la malla curricular y el discurso institucional también se visualiza en estos otros elementos curriculares. Por último, surge la necesidad de llevar a cabo estudios similares en programas de formación de docentes que se desempeñan en los niveles de enseñanza parvularia y secundaria. Esto permitiría contar con una visión más acabada respecto de los enfoques y puntos de encuentro/ desencuentro en torno a la educación inclusiva, así como también identificar necesidades formativas del profesorado que se está incorporando a un sistema educativo nacional cada día más diverso y que demanda transformaciones constantes. 


\section{Referencias}

Allday, R., Neilsen-Gatti, S., \& Hudson, T. (2013). Preparation for inclusion in teacher education pre-service curricula. Teacher Education and Special Education, 36(4), 298-311. http://dx.doi.org/10.1177/0888406413497485

Andreu, J. (2002). Las técnicas de análisis de contenido: una revisión actualizada. Sevilla: Fundación Centro de Estudios Andaluces.

Ávalos, B. (2010). Formación inicial docente en Chile: calidad y políticas. En C. Bellei, D. Contreras, y J. P. Valenzuela (Eds.), Ecos de la Revolución Pingüina: avances, debates y silencios en la reforma educacional (pp. 258284). Santiago de Chile: Unicef, Universidad de Chile.

Ávalos, B. (2014). La formación inicial docente en Chile: tensiones entre políticas de apoyo y control. Estudios Pedagógicos, 40, 11-28. http://dx.doi.org/10.4067/S0718-07052014000200002

Ávalos, B. y Matus, C. (2010). La formación inicial docente en Chile desde una óptica internacional. Informe nacional del estudio internacional IEA TEDS-M. Santiago de Chile: Mineduc.

Ávalos, B., Navarro, S., \& Téllez, F. (2010). Learning about the effectiveness of teacher education: A Chilean study. Perspectives in Education, 28(4), 11-21. Recuperado de: http://hdl.handle.net/11660/3178

Bellei, C. y Valenzuela, J. P. (2010). ¿Están las condiciones para que la docencia sea una profesión de alto estatus en Chile? En S. Martinic y G. Elacqua (Eds.), ¿Fin de ciclo? Cambios en la gobernanza del sistema educativo (pp. 257-283). Santiago de Chile: United Nations Educational, Scientific and Cultural Organization, Unesco, y Pontificia Universidad Católica de Chile.

Berner, H. y Bellei, C. (2011). Taller de análisis de politicas públicas. Tema del mes: educación. Santiago de Chile: Instituto de Asuntos Públicos, Universidad de Chile.

Blanton, L. \& Pugach, M. (2011). Using a classification system to probe the meaning of dual licensure in general and special education. Teacher Education and Special Education, 34(3), 219-234. http://dx.doi.org/10.1177/0888406411404569

Booth, T. \& Ainscow, M. (2011). Index for inclusion: Developing learning and participation in schools (3rd Edition). Bristol: Centre for Studies in Inclusive Education (CSIE). Recuperado de: http://dantescuola.gov.it/ dantescuola.eu/COMENIUS/Comenius2013_15/01Mobilita\%20in\%20 Bulgaria\%20-\%20Sofia/Readings/Index4INCLUSION.pdf 
48 FORMACIÓN INICIAL DOCENTE PARA LA EDUCACIÓN INCLUSIVA. ANÁLISIS DE TRES PROGRAMAS CHILENOS DE PEDAGOGÍA EN EDUCACIÓN BÁSICA QUE INCORPORAN LA PERSPECTIVA DE LA EDUCACIÓN INCLUSIVA - C. San Martín, C. Villalobos, C. Muñoz y I. Wyman

Cox, C., Meckes, L., y Bascopé, M. (2010). La institucionalidad formadora de profesores en Chile en la década del 2000: velocidad del mercado y parsimonia de las políticas. Pensamiento Educativo, 46, 205-245. Recuperado de: http://pensamientoeducativo.uc.cl/files/journals/2/ articles/468/public/468-1034-1-PB.pdf

Creswell, J. W. (2013). Research design: Qualitative, quantitative, and mixed methods approaches. London: Sage publications.

Damm, X. (2009). Representaciones y actitudes del profesorado frente a la integración de niños/as con necesidades educativas especiales al aula común. Revista Latinoamericana de Educación Inclusiva, 3(1), 25-35. Recuperado de: http://www.rinace.net/rlei/numeros/vol3-num1/art2.pdf.

Darling-Hammond, L. \& Baratz-Snowden, J. (2007). A good teacher in every classroom: Preparing the highly qualified teachers our children deserve. Educational Horizons, 85(2), 111-132. Recuperado de: http:// www.jstor.org/stable/42926597

De Jong, E. J. \& Harper, C. A. (2005). Preparing mainstream teachers for English-language learners: Is being a good teacher good enough? Teacher Education Quarterly, 32(2), 101-124. Recuperado de: http:// www.jstor.org/stable/23478724

DeLuca, C. (2012). Promoting inclusivity through and within teacher education Programmes. Journal of Education for Teaching, 38(5), 551569. http://dx.doi.org/10.1080/02607476.2013.739792

European Agency for Development in Special Needs Education. (2011). Teacher education for inclusion across Europe. Challenges and opportunities. Odense: Recuperado de: https://www.european-agency.org/sites/ default/files/te4i-challenges-and-opportunities_TE4I-SynthesisReport-EN.pdf

Farmer, T., Robinson, K., Elliott, S. J., \& Eyles, J. (2006). Developing and implementing a triangulation protocol for qualitative health research. Qualitative Health Research, 16(3), 377-394. http://dx.doi.org/10.1177/1049732305285708

Florian, L. (2007). Reimagining special education: The Sage handbook of special education. En L. Florian (Ed.), The Sage handbook of special education (pp. 7-20). London: Sage Publications. http://dx.doi.org/10.1080/13603111003778536

Florian, L. (2012). Preparing teachers to work in inclusive classrooms key lessons for the professional development of teacher educators from Scotland's inclusive practice project. Journal of Teacher Education, 63(4), 275-285. http://dx.doi.org/10.1177/0022487112447112 
Florian, L., Young, K., \& Rouse, M. (2010). Preparing teachers for inclusive and diverse educational environments: Studying curricular reform in an initial teacher education course. International Journal of Inclusive Education, 14(7), 709-722.

http://dx.doi.org/10.1080/13603111003778536

Forlin, C. (2012). Future directions for inclusive teacher education: An international perspective. London: Routledge. http://dx.doi.org/10.4324/9780203113585

Fullerton, A., Ruben, B., McBride, S., \& Bert, S. (2011). Development and design of a merged secondary and special education teacher preparation program. Teacher Education Quarterly, 38(2), 27-44. Recuperado de: http://www.jstor.org/stable/23479691

Glick, J., Yabiku, S., \& Bates, L. (julio, 2008). Instability and student achievement: Understanding the importance of school mobility, residential change and family disruption. Presentación realizada en la American Sociological Association Annual Meeting, Boston, MA.

Hardman, M. L. (2009). Redesigning the preparation of all teachers within the framework of an integrated program model. Teaching and Teacher Education, 25(4), 583-587. http://dx.doi.org/10.1016/j.tate.2009.02.005

Holland, D., Detgen, A., \& Gutekunst, L. (2008). Preparing elementary school teachers in the Southeast Region to work with students with disabilities. Report of the Southeast Regional Educational Laboratory at SERVE Center, Institute of Education Sciences, US Department of Education. Recuperado de: http://ies.ed.gov/ncee/edlabs/regions/ southeast/pdf/rel_2008065_main.pdf

Infante, M. (2010). Desafíos a la formación docente: inclusión educativa. Estudios Pedagógicos, 36(1), 287-297. http://dx.doi.org/10.4067/S0718-07052010000100016

Infante, M. \& Matus, C. (2009). Policies and practices on diversity: Reimagining possibilities for new discourses. Disability and Society, 24(4), 437-445. http://dx.doi.org/10.1080/09687590902879049

Ivankova, N. V., Creswell, J. W., \& Stick, S. L. (2006). Using mixed-methods sequential explanatory design: From theory to practice. Field methods, 18(1), 3-20. http://dx.doi.org/10.1177/1525822x05282260

Jackson, P. (1991). La vida en las aulas. Madrid: Morata.

Jiménez, F. y Montecinos, C. (en prensa) (2015). Diversidad, modelos de gestión y formación inicial docente: desafíos formativos desde una perspectiva de la justicia social. Revista Brasileira de Educação. 
50 FORMACIÓN INICIAL DOCENTE PARA LA EDUCACIÓN INCLUSIVA. ANÁLISIS DE TRES PROGRAMAS CHILENOS DE PEDAGOGÍA EN EDUCACIÓN BÁSICA QUE INCORPORAN LA PERSPECTIVA DE LA EDUCACIÓN INCLUSIVA - C. San Martín, C. Villalobos, C. Muñoz y I. Wyman

Jordan, A., Schwartz, E., \& McGhie-Richmond, D. (2009). Preparing teachers for inclusive classrooms. Teaching and Teacher Education, 25(4), 535542. http://dx.doi.org/10.1016/j.tate.2009.02.010

Krippendorff, K. W. (1990). Metodología de análisis de contenido: teoría y práctica. México: Paidós.

Lam, B. \& Tung Tsui, K. (2014). Curriculum mapping as deliberation examining the alignment of subject learning outcomes and course curricula. Studies in Higher Education, 41(8), 1-19. http://dx.doi.org/10.1080/03075079.2014.968539

Lewin, D. (2014). What's the use of ethical philosophy? The role of ethical theory in special educational needs. European Journal of Special Needs Education, 29(4), 536-547.

https://doi.org/10.1080/08856257.2014.933549

Lincoln, Y. S. \& Denzin, N. K. (1994). The fifth moment. En N. K Denzin \& Y. S. Lincoln (Eds.), The Handbook of qualitative research (1st edition) (pp. 575-586). London: Sage.

Liu, L. B. \& Milman, N. B. (2010). Preparing teacher candidates to teach diverse student populations through reflective practice. Reflective Practice, 11(5), 619-630.

http://dx.doi.org/10.1080/14623943.2010.516973

Melnick, S. L. \& Zeichner, K. M. (1995). Teacher education for cultural diversity: Enhancing the capacity of teacher education institutions to address diversity issues. Michigan: National Center for Research on Teacher Learning. Recuperado de: http://files.eric.ed.gov/fulltext/ED392751.pdf

Ministerio de Educación de Chile, Mineduc. (2005). Política Nacional de Educación Especial: nuestro compromiso con la diversidad. Recuperado de: http://www.mineduc.cl/biblio/documento/200704252105370. POLiTICAEDUCESP.pdf

Ministerio de Educación de Chile, Mineduc. (2007). Programa de educación intercultural bilingüe 2007. Recuperado de: http://www.mineduc.cl/ biblio/documento/200708240835400.ProgramadeEducaciOn.ppt

Ministerio de Educación de Chile, Mineduc. (2015). Diversificación de la enseñanza. Decreto Exento 83: Aprueba criterios y orientaciones de adecuación curricular para estudiantes con necesidades educativas especiales de educación parvularia y educación básica. Santiago de Chile: Autor. Recuperado de: http://www.educacionespecial.mineduc.cl/ usuarios/edu.especial/File/2015/Decreto\%2083-2015.pdf

Mizala, A., Hernández, T., y Makovec, M. (2011). Determinantes de la elección y deserción en la carrera de Pedagogía. Recuperado de: www.ciae.uchile. cl/download.php?file=2015-docentes/Determinantes.pdf 
Organization for Economic Cooperation and Development, OECD. (2010). Educating teachers for diversity: Meeting the challenge. Paris: OECD.

Pugach, M. C. \& Blanton, L. P. (2012). Enacting diversity in dual certification programs. Journal of Teacher Education, 63(4), 254-267. http://dx.doi.org/10.1177/0022487112446970

Román, M. \& Perticará, M. (2012). Student mobility in low quality schools: Segmentation among the most vulnerable students (Movilidad de estudiantes en escuelas de baja calidad histórica: Efectos en la segmentación entre los más vulnerables). Estudios de Economía, 39(2), 159. http://dx.doi.org/10.4067/s0718-52862012000200004

Rufinelli, A. (2013). La calidad de la formación inicial docente en Chile: la perspectiva de los profesores principiantes. Calidad en la educación, 39, 117-154. http://dx.doi.org/10.4067/s0718-45652013000200005

Sotomayor, C., Parodi, G., Coloma, C., Ibáñez, R., y Cavada, P. (2011). La formación inicial de docentes de Educación General Básica en Chile. ¿Qué se espera que aprendan los futuros profesores en el área de Lenguaje y Comunicación? Pensamiento Educativo, 48(1), 28-41. http:// dx.doi.org/10.7764/pel.48.1.2011.3

Tashakkori, A. \& Teddlie, C. (1998). Mixed methodology: Combining qualitative and quantitative approaches (Vol. 46). Thousand Oaks: Sage.

Tatto, M. T., Schwille, J., Senk, S., Ingvarson, L., Rowley, G., Peck, R., Bankov, K., ... Reckase, M. (2012). Policy, practice, and readiness to teach primary and secondary Mathematics in 17 countries. Findings from the IEA teacher education and development study in Mathematics (TEDS-M). Recuperado de: http://www.iea.nl/fileadmin/user_upload/ Publications/Electronic_versions/TEDS-M_International_Report.pdf

Taylor, S. J. y Bogdan, R. (1987). Introducción a los métodos cualitativos de investigación. Buenos Aires: Paidós.

Tenorio, S. (2005). La integración escolar en Chile: perspectiva de los docentes sobre su implementación. Revista Electrónica Iberoamericana sobre Calidad, Eficacia y Cambio en Educación, 3(1), 823-831. Recuperado de: http://www.ice.deusto.es/RINACE/reice/Vol3n1_e/Tenorio.pdf

Tenorio, S. (2011). Formación inicial docente y necesidades educativas especiales. Estudios Pedagógicos, 37(2), 249-267. http://dx.doi.org/10.4067/s0718-07052011000200015

Toledo, G. y Valenzuela, J. P. (2012). Ordenamiento de profesores y estudiantes entre y dentro de los establecimientos escolares: el caso de Chile. Universidad de Chile, Departamento de Economía. Santiago de Chile: Centro de Investigación Avanzada en Educación (CIAE). Recuperado 
52 FORMACIÓN INICIAL DOCENTE PARA LA EDUCACIÓN INCLUSIVA. ANÁLISIS DE TRES PROGRAMAS CHILENOS DE PEDAGOGÍA EN EDUCACIÓN BÁSICA QUE INCORPORAN LA PERSPECTIVA DE LA EDUCACIÓN INCLUSIVA - C. San Martín, C. Villalobos, C. Muñoz y I. Wyman

de: http://www.ciae.uchile.cl/download.php?file=2015.docentes/ Ordenamiento_profesores.pdf.

United Nations Educational, Scientific and Cultural Organization, Unesco. (2009). Policy guidelines on inclusion in education. Paris: Unesco. Recuperado de: http://unesdoc.unesco.org/ images/0017/001778/177849e.pdf

Varas, L., Felmer, P., Gálvez, G., Lewin, R., Martínez, C., Navarro, S., ... Schwarze, G. (2008). Oportunidades de preparación para enseñar matemática de futuros profesores de Educación General Básica en Chile. Calidad en la Educación, 29, 64-88. Recuperado de: http:// www.cned.cl/public/secciones/seccionrevistacalidad/doc/60/cse_ articulo740.pdf

Venegas, C. (2013). Hacia la innovación en la formación inicial docente para un desempeño exitoso en contextos de alta vulnerabilidad social y educativo. Revista de Estudios y Experiencias en Educación, 12(23), 47-59. Recuperado de: http://www.redalyc.org/articulo.oa?id=243128148003

Villalobos, C., San Martín, C., y Wyman, I. (2016). Formación inicial de profesores y educación inclusiva: un análisis de las características de los programas de formación inicial de docentes de educación básica en Chile (Documento de trabajo,) Universidad Diego Portales. Santiago de Chile: Universidad Diego Portales

Zapata, R. (2011). Formación del futuro profesorado en diversidad cultural: estudio de un caso en el contexto universitario chileno. Revista Digital Akadèmeia, 2(2). Recuperado de: http://webcache.googleusercontent. com/search?q=cache:zueVOuMik1YJ:www.revistaakademeia. $\mathrm{cl} / \% 3 F p \% 3 D 727 \&$ num $=1$ \&hl=es-419\&gl=cl\&strip=0\&vwsrc $=0$

Recibido: 19/08/2016

Aceptado: 01/06/2017 